\title{
Suppression of CD51 in pancreatic stellate cells inhibits tumor growth by reducing stroma and altering tumor-stromal interaction in pancreatic cancer
}

\author{
KOHEI HORIOKA ${ }^{1}$, KENOKI OHUCHIDA ${ }^{1,2}$, MASAFUMI SADA $^{1}$, BIAO ZHENG ${ }^{1}$, TAIKI MORIYAMA ${ }^{1}$, \\ HAYATO FUJITA $^{1}$, TATSUYA MANABE ${ }^{1}$, TAKAO OHTSUKA ${ }^{1}$, MASAYA SHIMAMOTO ${ }^{3}$, \\ TETSUYUKI MIYAZAKI $^{1,4}$, KAZUHIRO MIZUMOTO ${ }^{3}$, YOSHINAO ODA ${ }^{4}$ and MASAFUMI NAKAMURA ${ }^{1}$ \\ Departments of ${ }^{1}$ Surgery and Oncology, ${ }^{2}$ Advanced Medical Initiatives, Graduate School of Medical Sciences, \\ Kyushu University; ${ }^{3}$ Kyushu University Hospital Cancer Center; ${ }^{4}$ Department of Anatomic Pathology, \\ Graduate School of Medical Sciences, Kyushu University, Fukuoka, Japan
}

Received November 30, 2015; Accepted January 5, 2016

DOI: 10.3892/ijo.2016.3374

\begin{abstract}
Pancreatic stellate cells (PSCs) enhance the malignant behavior of pancreatic cancer by interacting with cancer cells and producing extracellular matrix (ECM). To date, several stroma-targeted therapies for pancreatic cancer have been attempted, but these therapies are still not in practical use. Integrins expressed in stromal cells are involved in fibrosis of several organs, as well as promoting tumor malignancy. We investigated whether CD51, also known as integrin $\alpha \mathrm{V}$, expressed in PSCs was associated with stromal formation of pancreatic cancer and enhancement of tumor malignancy. We also assessed the effects of suppression of CD51 in PSCs on pancreatic cancer. Immunohistochemistry for CD51 in resected pancreatic cancer tissues showed that high expression of CD51 in the tumor stroma was associated with lymph node metastasis $(\mathrm{P}=0.025)$, positive pathologic margin $(\mathrm{P}=0.025)$, and shorter patient survival times $(\mathrm{P}=0.043)$. Lentivirus-mediated short hairpin RNA knockdown of CD51 decreased the proliferation and migration of PSCs. Quantitative real-time polymerase chain reaction showed that expression levels of genes related with ECM and tumor-stromal interactions were decreased by CD51 knockdown in PSCs. In a co-implantation model of pancreatic cancer cells and PSCs, tumor growth in vivo was inhibited by CD51 knockdown in PSCs $(\mathrm{P}<0.05)$. We also found reduced tumor stroma and decreased proliferation of cancer cells in implanted cancer tissues with CD51-silenced PSCs $(\mathrm{P}<0.05)$. Our results showed that CD51 expression in
\end{abstract}

Correspondence to: Dr Kenoki Ohuchida, Department of Surgery and Oncology, Graduate School of Medical Sciences, Kyushu University, 3-1-1 Maidashi, Fukuoka 812-8582, Japan

E-mail:kenoki@med.kyushu-u.ac.jp

Key words: CD51, pancreatic cancer, pancreatic stellate cells, stroma, tumor-stromal interaction pancreatic cancer stroma is associated with enhanced tumor malignancy and that CD51 may be a potential therapeutic target for pancreatic cancer.

\section{Introduction}

Pancreatic cancer is one of the most aggressive cancers, representing the fourth leading cause of cancer-related death in the United States (1). Despite recent advances in multimodal therapeutic approaches including surgery and chemoradiotherapy, the fatal prognosis of pancreatic cancer has remained unchanged over the last few decades.

Remarkable desmoplasia is one of the pathological features of pancreatic cancer and contributes to the enhancement of its malignant potential (2). Desmoplasia involves an excessive amount of extracellular matrix (ECM) proteins, which prevent drug delivery to cancer cells, leading to chemoresistance (3). To date, several therapeutic agents have been developed to reduce excessive ECM in combination with chemotherapeutics, but these agents have not yet been applied in practical use $(4,5)$.

Pancreatic stellate cells (PSCs) are major sources of ECM production in desmoplasia. In the normal pancreas, PSCs are located in close proximity of pancreatic acinar cells $(6,7)$. During pancreatic injury and inflammation, resident PSCs transform into a myofibroblastic phenotype and secrete excessive amounts of ECM proteins, leading to desmoplasia (8). In pancreatic cancer, carcinoma cells stimulate PSCs to produce ECM proteins and many cytokines. Conversely, PSCs also stimulate pancreatic cancer cells to enhance cancer cell proliferation, motility, invasion and chemoresistance $(9,10)$.

Integrins are heterodimeric cell surface receptors that mediate adhesion to the ECM and immunoglobulin superfamily molecules. At least 24 distinct integrin heterodimers are formed by the combination of $18 \alpha$-subunits and eight $\beta$-subunits (11). Integrins regulate various functions in tumor cells, including migration, invasion, proliferation, and survival (11). In several solid tumors, the expression of particular integrins correlates with increased disease progression and decreased patient survival (12-14). Integrins are also 
expressed on stromal cells in the tumor microenvironment, such as vascular endothelial cells (15), fibroblasts (16), and bone marrow-derived cells $(17,18)$, and contribute to enhance tumor progression. For example, expression of $\alpha 11 \beta 1$ on fibroblasts in non-small cell lung carcinoma increased tumor growth by stimulating the release of insulin-like growth factor 2 (16). CD51, also known as integrin $\alpha \mathrm{V}$, heterodimerizes with $\beta 1, \beta 3, \beta 5, \beta 6$, and $\beta 8$ integrins and interacts with several ECM proteins to function in various physiological roles such as cell adhesion and migration (19). Furthermore, CD51 functions as a receptor for various growth factors such as TGF- $\beta 1$ and mediates some biological signaling $(20,21)$. A recent study showed that CD51 in hepatic stellate cells activates latent TGF- $\beta 1$ embedded in the ECM, leading to hepatic fibrosis (21). On the other hand, CD51 in cancer cells is thought to be associated with cancer progression in some solid tumors $(22,23)$. However, little is known about the role of CD51 in pancreatic cancer.

In the present study, we investigated whether CD51 in PSCs correlated with the formation of tumor desmoplasia and cancer progression to assess the significance of CD51 in pancreatic cancer.

\section{Materials and methods}

Patients and pancreatic tissues. Pancreatic cancer tissues were obtained from 94 patients who underwent surgical resection for pancreatic cancer at our institution. Survival was measured from the time of pancreatic resection until death. Prognosis was determined in June 2015. The median overall survival time was 19.5 months (range, 1-166 months). Seventy patients died during follow-up. The study was approved by the Ethics Committee of Kyushu University and conducted according to the Ethical Guidelines for Human Genome/ Gene Research enacted by the Japanese Government and the Helsinki Declaration.

Immunohistochemical procedures and evaluation of sections. Tissues were sectioned to a thickness of $4 \mu \mathrm{m}$ and were incubated with rabbit monoclonal anti-CD51 antibody (ab179475; 1:500; Abcam) overnight at $4^{\circ} \mathrm{C}$ and subsequently incubated with biotin-free horseradish peroxidase enzyme-labeled polymer (Envision Plus System, Dako) for $40 \mathrm{~min}$ at room temperature. The labeled antigens were visualized using 3,3'-diaminobenzidine tetrahydrochloride as a chromogen. Counterstaining was performed with hematoxylin.

In vivo tumor tissues were immunostained with the following primary antibodies: mouse monoclonal anti- $\alpha-S M A$ (1:100; Dako), rabbit polyclonal anti-cytokeratin 19 (CK19; sc-25724; 1:50; Santa Cruz Biotechnology) and rabbit polyclonal anti-proliferating cell nuclear antigen (PCNA; ab2426; 1:2,000; Abcam).

Nerve fibers readily express CD51 and thus were used as internal controls for CD51 expression. The distribution of CD51 staining was evaluated by the proportion of stained cells in the stroma or cancer cells, and scored as follows: 0 , $0 \% ; 1,<25 \% ; 2,26-50 \% ; 3,51-75 \%$; or $4,76-100 \%$. Similarly, the staining intensity was scored as follows: 0 , no staining; 1 , weak staining; 2 , moderate staining; or 3 , strong staining. We calculated the final score by multiplying the proportional score by the intensity score and then divided samples into two groups: CD51-high (final score $>5$ ) and CD51-low (final score <5).

Immunofluorescence staining. PSCs $\left(1 \times 10^{4}\right.$ cells) were plated on a glass-bottom 24-well dish (MatTek Corporation, Ashland, MA, USA) and incubated for $24 \mathrm{~h}$. PSCs were then fixed with methanol, blocked with $3 \%$ bovine serum albumin in phosphate-buffered saline and incubated with mouse anti- $\alpha$-SMA (1:100; Dako) and rabbit anti-CD51 antibodies (ab179475; 1:500; Abcam) for $2 \mathrm{~h}$ at room temperature. The cells were then incubated for $1 \mathrm{~h}$ with Alexa 488-conjugated anti-rabbit IgG (1:200; Life Technologies Corporation) and Alexa 594-conjugated anti-mouse IgG (1:200; Life Technologies Corporation). Nuclear DNA was counterstained with 4',6-diamidino2-phenylindole. Biorevo BZ-9000 (Keyence) was used for immunofluorescence microphotography.

Sirius red staining and measurements. To assess the amount of collagen fiber in tumor stroma, sections were stained using a Picrosirius Red Staining kit (Polysciences Inc.) according to the manufacturer's instructions. The Sirius red-stained area was measured using Adobe Photoshop Element (Adobe Systems Inc.) by selecting stained fibers in three fields at a magnification of x200 under a light microscope.

Cell isolation and culture conditions. We isolated human PSCs from pancreatic cancer surgical specimens and adjacent normal pancreatic tissues using the outgrowth method described by Bachem and colleagues (7). Cells were maintained as previously described (24). We confirmed that the PSCs exhibited a fibroblast-like morphology and were immunohistochemically positive for $\alpha$-SMA. All established PSCs were used between passages 3 and 8 . Normal human pancreatic epithelial cells were purchased from DS Pharma Biomedical. We also used 12 human pancreatic cell lines: Capan-1, Capan-2, ASPC-1, CFPAC-1, BxPC3, PANC-1, Hs766T, KP-2, KP-3, SUIT-2, MIAPaCa-2, and SW1990 cells. All cell lines were propagated and frozen immediately after arrival. The cells revived from the frozen stock were used within 3 months. Cell lines were regularly authenticated and matched short tandem repeat DNA profiles of the original cell lines by JCRB. The cells were maintained as previously described (24).

Establishment of immortalized PSCs. We cloned the DNA encoding hTERT and SV40 LargeT in the pLVSIN vector. We used these vectors to construct lentiviral particles for infection of human PSCs, followed by G418 selection.

shRNA-mediated knockdown of CD51 in PSCs. High-titer lentiviral particles expressing shRNA against CD51 were obtained from Sigma (MISSION Lentiviral Transduction Particles; shCD51-1, TRCN0000010769; shCD51-2, TRCN0000342484). GIPZ Non-silencing Lentiviral shRNA Control (Thermo Scientific) was used as a control (shControl). Transfection into PSCs was performed, followed by puromycin selection. Knockdown efficacy was confirmed by quantitative real-time reverse-transcription polymerase chain reaction (qRT-PCR) and western blotting. 
Table I. Primers used for quantitative RT-PCR.

\begin{tabular}{lll}
\hline Primer & \multicolumn{1}{c}{ Forward sequence 5'-3' } & \multicolumn{1}{c}{ Reverse sequence 5'-3' } \\
\hline CD51 & AAACTCGCCAGGTGGTATGTGA & CTGGTGCACACTGAAACGAAGA \\
$\alpha$-SMA & GACAATGGCTCTGGGCTCTGTAA & CTGTGCTTCGTCACCCACGTA \\
Collagen I & TCTAGACATGTTCAGCTTTGTGGAC & TCTGTACGCAGGTGATTGGTG \\
Fibronectin & ACAGAACTATGATGCCGACCAGAAG & ACTGATCTCCAATGCGGTACATGA \\
Periostin & GCCCAATTAGGCTTGGCATC & GTTTCCAGTATTTGCCCGTGTA \\
Osteopontin & CATCAGACTGGTGAGAATCATC & ATCTCCTAGCCCCACAGAAT \\
CTGF & GTGTGTGACGAGCCCAAGGA & GGTCTGGGCCAAACGTGTCT \\
VEGF-A & TCACAGGTACAGGGATGAGGACAC & CAAAGCACAGCAATGTCCTGAAG \\
PDGF-A & CACTAAGCATGTGCCCGAGAA & CGTAAATGACCGTCCTGGTCTTG \\
PDGF-B & CAGCCACGACTGCCATGTAA & ACCTACATCTGCCAGAAATTGTCA \\
SDF-1 & GAGCCAACGTCAAGCATCTCAA & TTAGCTTCGGGTCAATGCACAC \\
GAPDH & GCACCGTCAAGGCTGAGAAC & TGGTGAAGACGCCAGTGGA \\
\hline
\end{tabular}

Cell proliferation. Cells $\left(1 \times 10^{3}\right)$ were seeded in triplicate in a 96-well plate in a $100 \mu \mathrm{l}$ volume of Dulbecco's modified Eagle's medium (DMEM) containing $10 \%$ fetal bovine serum (FBS). At day $0,1,2$, and 3, ATP was measured using the CellTiter-Glo Luminescent Cell Viability Assay (Promega) according to the manufacturer's instructions.

Cell migration assay. Cell migration was measured by counting the number of cells that migrated through transwell chambers with $8-\mu \mathrm{m}$ pores (BD Biosciences). PSCs $\left(5 \times 10^{4}\right.$ cells) were resuspended in $250 \mu \mathrm{l}$ of DMEM containing $10 \% \mathrm{FBS}$ and were placed in the upper chamber. After incubation for $24 \mathrm{~h}$, the migrated cells were fixed with $70 \%$ ethanol, stained with hematoxylin and eosin (H\&E), and counted in five random fields at a magnification of x 200 under a light microscope. The results are expressed as the mean number of migrated cells per field. Each experiment was carried out in triplicate and repeated twice.

Quantitative real-time reverse-transcription polymerase chain reaction ( $q R T-P C R$ ). Total RNA was extracted from cultured cells using a High Pure RNA Isolation kit (Roche Diagnostics, Mannheim, Germany) and DNase I (Roche Diagnostics) treatment according to the manufacturer's instructions. qRT-PCR was performed using iTaq Universal SYBR Green One-Step Kit, iScript reverse transcriptase, and a CFX96 Touch RealTime PCR Detection System (Bio-Rad Laboratories). Primers for CD51, $\alpha$-SMA, collagen I, fibronectin, periostin, connective tissue growth factor (CTGF), TGF- $\beta 1$, vascular endothelial growth factor-A (VEGF-A), platelet-derived growth factor (PDGF)-A, -B, stromal cell-derived factor-1 (SDF-1), and GAPDH were purchased from Takara Bio Inc. (Tokyo, Japan). We designed specific primers for osteopontin using primer 3 . Data are presented as relative expression normalized to GAPDH levels. The primer sequences are shown in Table I.

Western blotting. Protein was extracted from PSCs using PRO-PREP (iNtRON biotechnology) according to the manufacturer's instructions. Cellular protein $(20 \mu \mathrm{g})$ was fractionated on a $4 \%$ to $15 \%$ Mini-Protean TGX Precast Gel (Bio-Rad Laboratories) and transferred to Trans-Blot Turbo Mini PVDF Transfer Packs (Bio-Rad Laboratories) using the Trans-Blot Turbo Transfer Starter System (Bio-Rad Laboratories). The membrane was incubated overnight at $4^{\circ} \mathrm{C}$ with anti-CD51 (ab179475; 1:500; Abcam) or anti- $\beta$-actin (ab8227; 1:5,000; Abcam) antibodies and then probed with horseradish peroxidase-conjugated secondary antibodies (Cell Signaling Technology). Immunoblots were detected by enhanced chemiluminescence with a ChemiDoc XRS System (Bio-Rad Laboratories).

In vivo experiments. To analyze the effects of CD51 in PSCs on tumor growth in vivo, SUIT-2 cells $\left(1 \times 10^{6}\right)$ suspended in $100 \mu 1$ DMEM with or without PSCs $\left(1 \times 10^{6}\right)$ were subcutaneously transplanted into the back of 7-week-old female nude mice ( $n=10$ per each group). The mice were sacrificed at day 42, and all subcutaneous tumors were excised and weighed. Tumor volume was calculated using the following formula: $\pi / 6 \times(\mathrm{L} \times \mathrm{W} \times \mathrm{W})$, where $\mathrm{L}$ is the largest tumor diameter and $\mathrm{W}$ the smallest tumor diameter. All mouse experiments were approved by the Ethics Committee of Kyushu University.

Statistical analysis. A $\chi^{2}$ test was used to analyze the correlation between CD51 expression and clinicopathological characteristics. Survival analysis was undertaken using Kaplan-Meier analysis and curves were compared using the log-rank test. For the in vitro experiments, results are expressed as means \pm standard deviation. Comparisons between groups were performed using the Student's t-test. Values of $\mathrm{P}<0.05$ were considered statistically significant in all analyses. The statistical analyses were conducted using JMP Pro 11 software (SAS Institute).

\section{Results}

CD51 expression in pancreatic cancer. To investigate CD51 expression in pancreatic cancer, we performed immunohistochemistry for CD51 using resected pancreatic cancer specimens. CD51 was expressed in both the tumor stroma and 
A

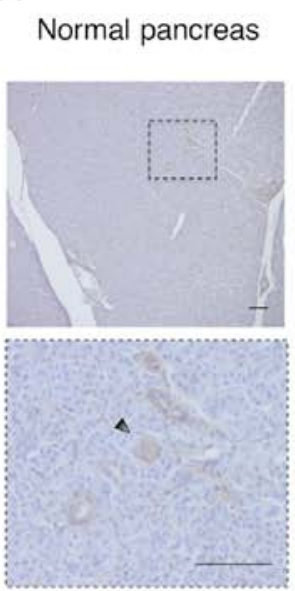

B

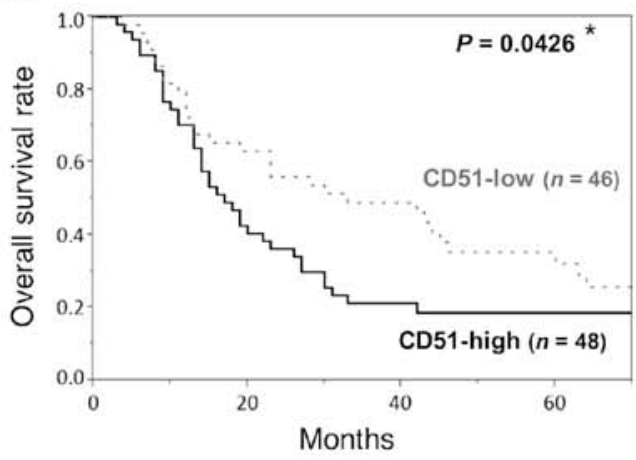

Stroma - high $(n=48)$
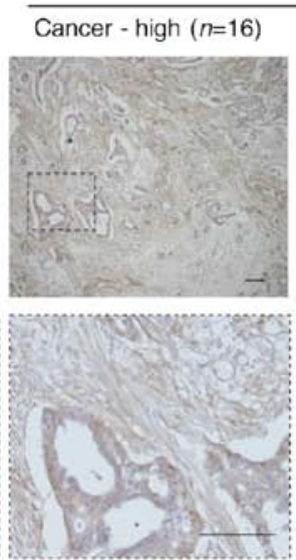

Cancer - low $(n=32)$
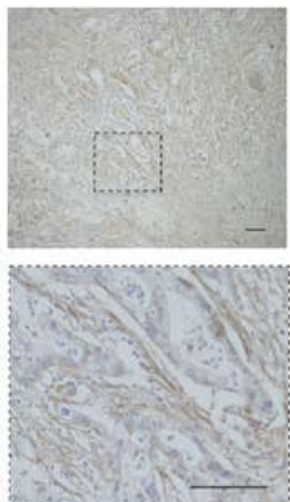

Stroma - low ( $n=46)$

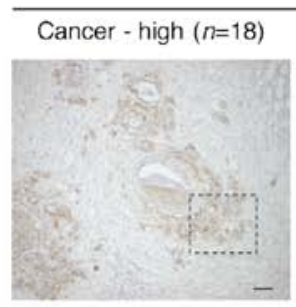

Cancer - low $(n=28)$
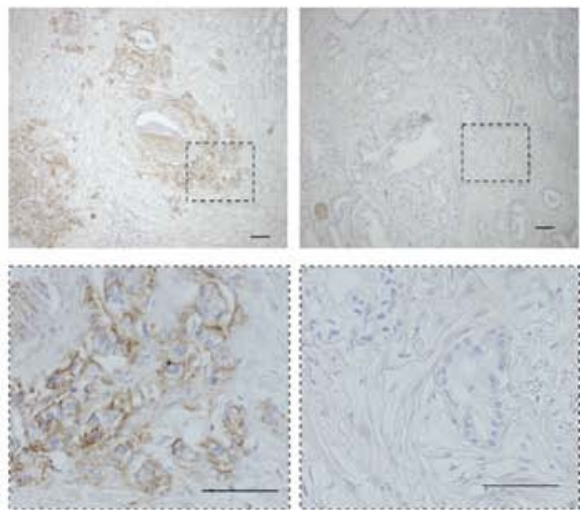

C

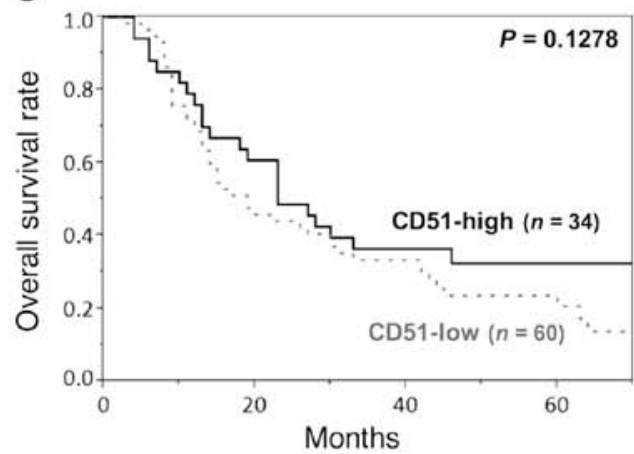

D

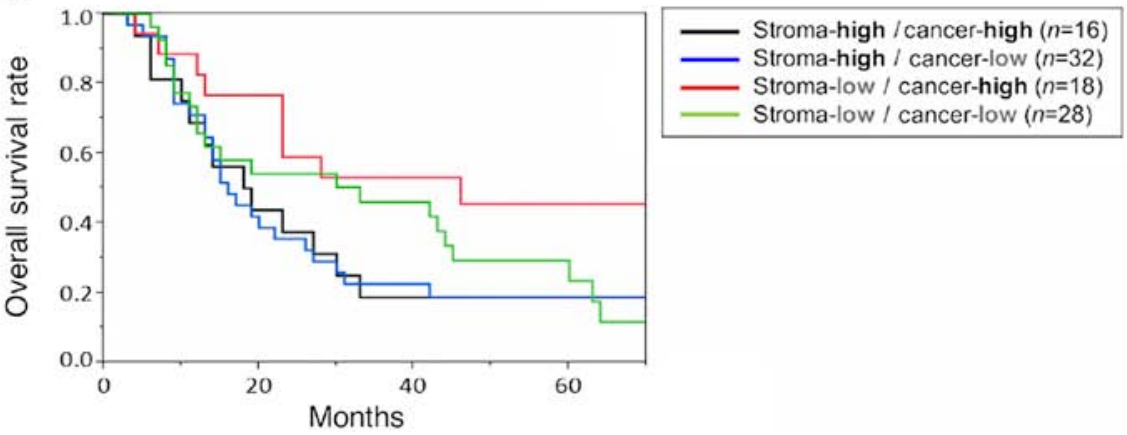

Figure 1. CD51 expression in pancreatic cancer. (A) Immunohistochemistry of CD51 in normal pancreas and pancreatic cancer. CD51 was slightly expressed in pancreatic ducts and stroma in normal pancreas. Nerve fibers (arrowhead) were used as positive internal controls. Expression levels of CD51 in the stroma and cancer cells were divided into two groups, high and low, according to the intensity and distribution. Original magnification: $\mathrm{x} 100$ (top). High magnification images are shown in the lower panel. Scale bars, $100 \mu \mathrm{m}$. (B) Kaplan-Meier survival analysis of CD51 expression in the stroma of pancreatic cancer. CD51-high expression in the stroma was associated with shorter patient survival times $(\mathrm{P}<0.05)$. (C) Kaplan-Meier survival analysis of CD51 expression in cancer cells of pancreatic cancer. CD51 expression in cancer cells was not correlated with patient survival time (P=0.1278). (D) Kaplan-Meier survival analysis of CD51 expression in both the stroma and cancer cells of pancreatic cancer. In the four subgroups classified by the expression levels of CD51 in both the stroma and cancer cells, no relationship with patient survival was observed.

cancer cells, while in the adjacent normal pancreas, CD51 was slightly expressed in the stroma and pancreatic duct (Fig. 1A). Pancreatic tumor samples were divided into four subgroups according to the staining intensity and distribution of CD51 in the stroma and cancer cells (Fig. 1A). Correlation between CD51 expression in the stroma or cancer cells and clinicopathologic characteristics is shown in Tables II and III. Confining analysis to stromal CD51 expression, lymph node metastasis $(\mathrm{P}=0.025)$ and positive pathologic margin $(\mathrm{P}=0.025)$ were observed more frequently in the CD51-high group than in the CD51-low group (Table II). On the contrary, in the cancer cells, lymph node metastasis $(\mathrm{P}=0.043)$ and $\mathrm{G} 3$ grade tumor $(\mathrm{P}=0.040)$ were observed more frequently in the CD51-high group than in the CD51-low group (Table III). These results suggest that CD51 expression in both the stroma and cancer cells is associated with tumor malignancy, including lymph node metastasis and cancer cell invasiveness.

Stromal CD51 expression is associated with shorter patient survival time. Stromal CD51 expression was associated with shorter patient survival time (Fig. 1B). The median overall survival times for stromal CD51-high and CD51-low 
Table II. Relationship between stromal CD51 expression and various clinicopathological factors.

\begin{tabular}{|c|c|c|c|}
\hline \multirow[b]{2}{*}{ Characteristics } & \multicolumn{2}{|c|}{ Stromal CD51 expression } & \multirow[b]{2}{*}{ P-value } \\
\hline & $\begin{array}{c}\text { High, } \\
\mathrm{n}=48(\%)\end{array}$ & $\begin{array}{c}\text { Low, } \\
\mathrm{n}=46(\%)\end{array}$ & \\
\hline Age (years) & & & 0.424 \\
\hline$\geq 65$ & $32(67)$ & 27 (59) & \\
\hline$<65$ & $16(33)$ & $19(41)$ & \\
\hline pT category & & & 0.062 \\
\hline $\mathrm{pT} 1 / \mathrm{pT} 2$ & $2(4)$ & $7(15)$ & \\
\hline pT3/pT4 & $46(96)$ & $39(85)$ & \\
\hline Lymph node metastasis & & & $0.025^{\mathrm{a}}$ \\
\hline No & $8(17)$ & $17(36)$ & \\
\hline Yes & $40(83)$ & $29(63)$ & \\
\hline UICC stage & & & 0.096 \\
\hline I & $1(2)$ & $6(13)$ & \\
\hline II & $45(94)$ & $39(85)$ & \\
\hline III/IV & $2(4)$ & $1(2)$ & \\
\hline Histologic grade & & & 0.988 \\
\hline $\mathrm{G} 1 / \mathrm{G} 2$ & $31(65)$ & $29(63)$ & \\
\hline G3 & $15(31)$ & $15(33)$ & \\
\hline Others & $2(4)$ & $2(4)$ & \\
\hline Pathologic margin & & & $0.025^{\mathrm{a}}$ \\
\hline Negative & $26(54)$ & $35(76)$ & \\
\hline Positive & $22(46)$ & $11(24)$ & \\
\hline Perilymphatic invasion & & & 0.083 \\
\hline No & $10(21)$ & $17(37)$ & \\
\hline Yes & 38 (79) & $29(63)$ & \\
\hline Perivascular invasion & & & 0.062 \\
\hline No & $14(29)$ & $22(48)$ & \\
\hline Yes & $34(71)$ & $24(52)$ & \\
\hline Perineural invasion & & & 0.211 \\
\hline No & $5(10)$ & $9(20)$ & \\
\hline Yes & $43(90)$ & $37(80)$ & \\
\hline
\end{tabular}

UICC, International Union Against Cancer.

groups were 17 and 33 months, respectively. However, CD51 expression in cancer cells was not associated with shorter patient survival time (Fig. 1C). Furthermore, we observed no correlation with patient survival among the four subgroups categorized by CD51 expression in the stroma and cancer cells (Fig. 1D).

Knockdown of CD51 suppresses the proliferation and migration of PSCs. In pancreatic cancer, PSCs are thought to be major cellular constituents in tumor stroma. Therefore, we next investigated CD51 expression in human PSCs isolated from resected pancreatic cancer tissues. qRT-PCR showed that several kinds of PSCs expressed CD51 at variable levels,
Table III. Relationship between CD51 expression in cancer cells and various clinicopathological factors.

\begin{tabular}{|c|c|c|c|}
\hline \multirow[b]{2}{*}{ Characteristics } & \multicolumn{2}{|c|}{$\begin{array}{l}\text { CD51 expression } \\
\text { in cancer cells }\end{array}$} & \multirow[b]{2}{*}{ P-value } \\
\hline & $\begin{array}{c}\text { High, } \\
\mathrm{n}=34(\%)\end{array}$ & $\begin{array}{c}\text { Low, } \\
\mathrm{n}=60(\%)\end{array}$ & \\
\hline Age (years) & & & 0.769 \\
\hline$\geq 65$ & $22(65)$ & $37(62)$ & \\
\hline$<65$ & $12(35)$ & $23(38)$ & \\
\hline pT category & & & 0.851 \\
\hline $\mathrm{pT} 1 / \mathrm{pT} 2$ & $3(9)$ & $6(10)$ & \\
\hline $\mathrm{pT} 3 / \mathrm{pT} 4$ & $31(91)$ & $54(90)$ & \\
\hline Lymph node metastasis & & & $0.043^{\mathrm{a}}$ \\
\hline No & $5(15)$ & $20(33)$ & \\
\hline Yes & $29(85)$ & $40(67)$ & \\
\hline UICC stage & & & 0.927 \\
\hline I & $3(9)$ & $4(30)$ & \\
\hline II & $30(88)$ & $54(90)$ & \\
\hline III/IV & $1(3)$ & $2(3)$ & \\
\hline Histologic grade & & & $0.040^{\mathrm{a}}$ \\
\hline $\mathrm{G} 1 / \mathrm{G} 2$ & $16(47)$ & $44(74)$ & \\
\hline G3 & $16(47)$ & $14(23)$ & \\
\hline Others & $2(6)$ & $2(3)$ & \\
\hline Pathologic margin & & & 0.673 \\
\hline Negative & $23(68)$ & $38(63)$ & \\
\hline Positive & $11(32)$ & $22(37)$ & \\
\hline Perilymphatic invasion & & & 0.715 \\
\hline No & $9(26)$ & $18(30)$ & \\
\hline Yes & $25(74)$ & $42(70)$ & \\
\hline Perivascular invasion & & & 0.370 \\
\hline No & $11(32)$ & $25(42)$ & \\
\hline Yes & $23(68)$ & $35(58)$ & \\
\hline Perineural invasion & & & 0.251 \\
\hline No & $7(21)$ & $7(12)$ & \\
\hline Yes & $27(79)$ & $53(88)$ & \\
\hline
\end{tabular}

UICC, International Union Against Cancer.

and similar tendencies were observed in PSCs that were isolated from normal pancreas adjacent to tumor and also in pancreatic cancer cell lines (Fig. 2A). On the contrary, CD51 was not expressed in normal pancreatic epithelial cells (Fig. 2A). Immunofluorescence staining demonstrated that most PSCs expressing $\alpha$-SMA, a marker for activated PSCs, also expressed CD51 (Fig. 2B).

To clarify the functional role of CD51 in PSCs, we performed shRNA-mediated knockdown of CD51 in immortalized human PSCs (Fig. 2C and D). Knockdown of CD51 led to suppression of the proliferation and migration of PSCs (Fig. 2E and F). 
A
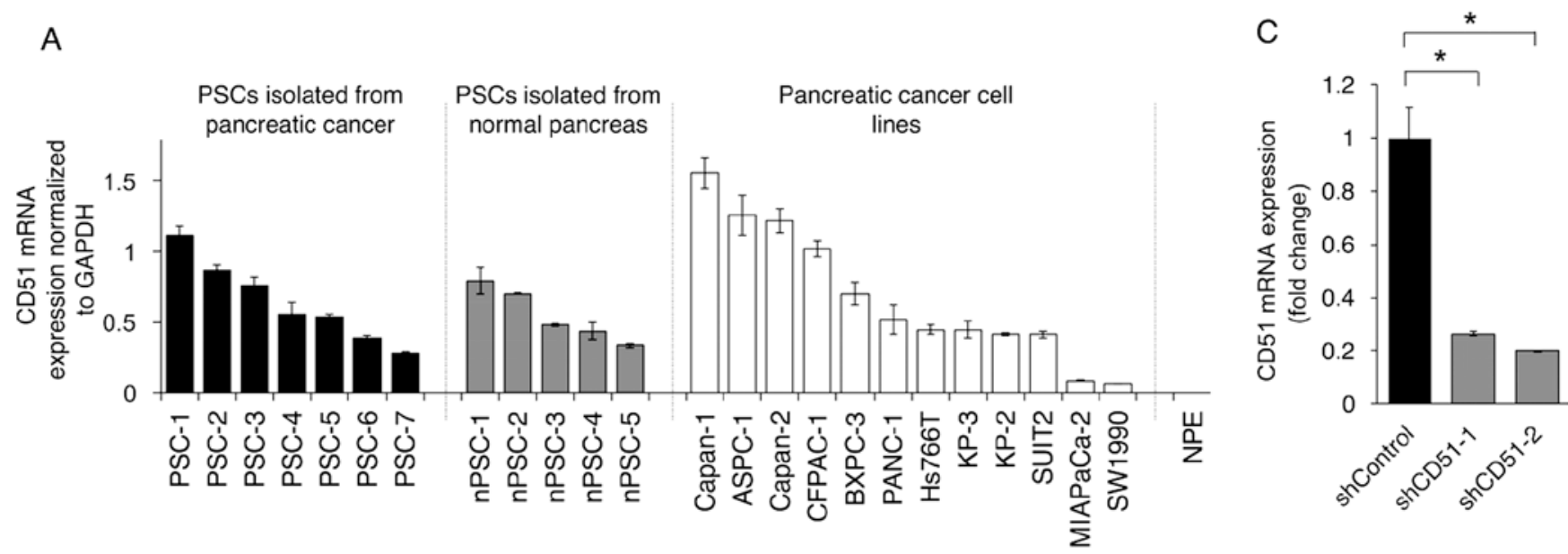

B
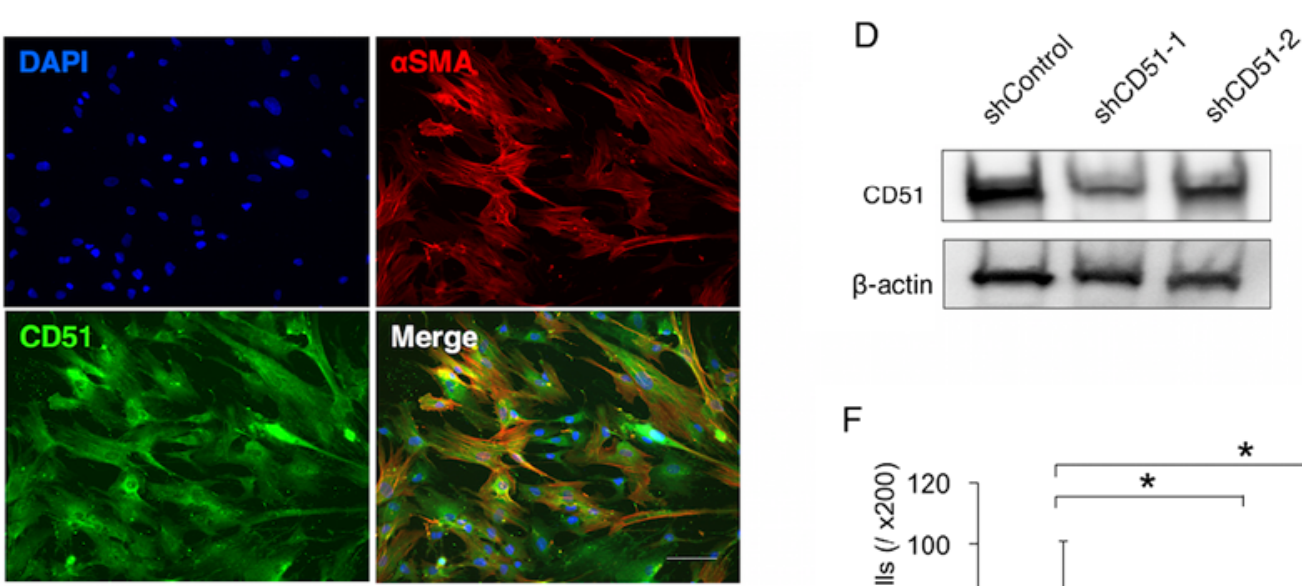

E
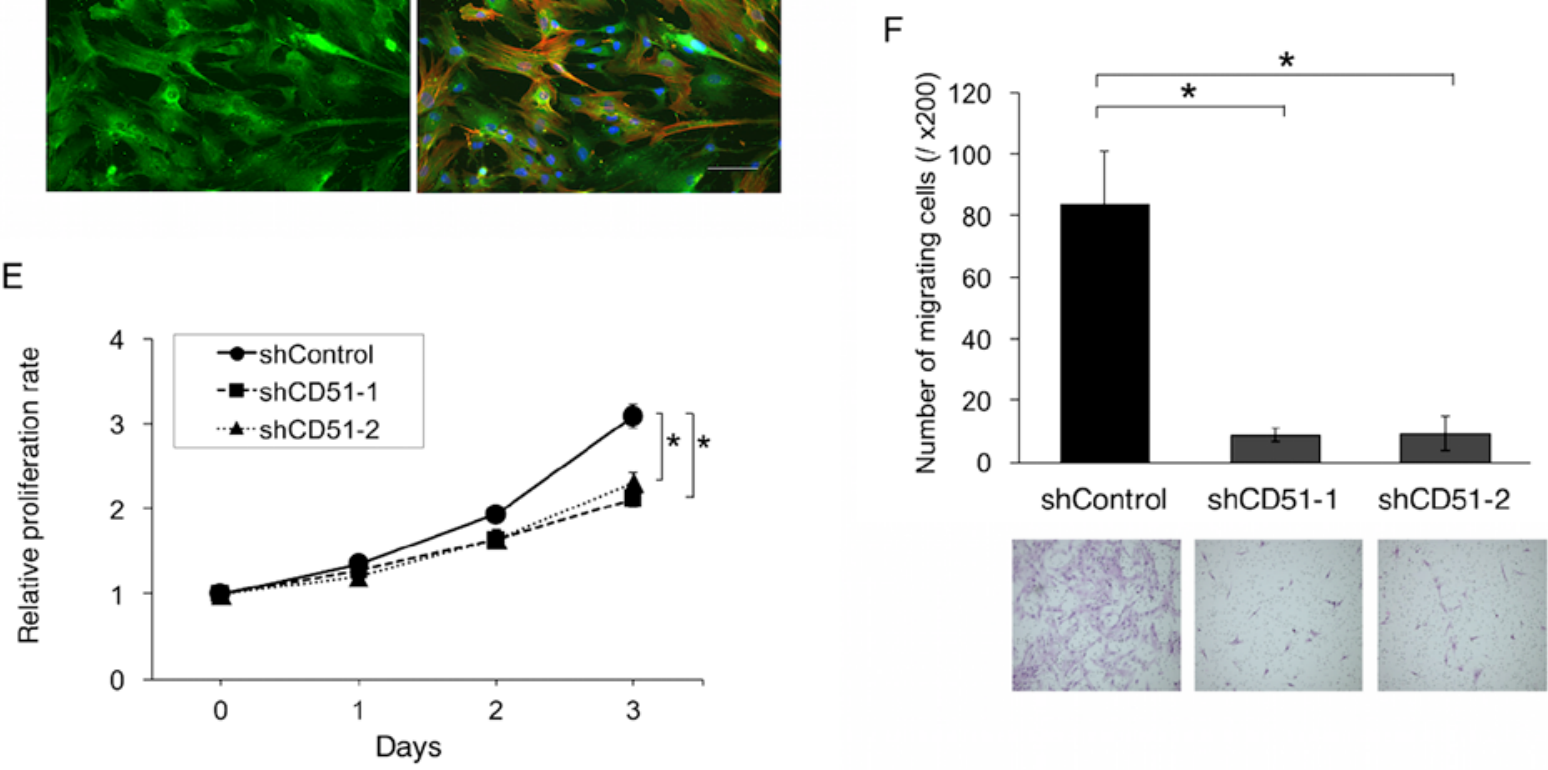

Figure 2. Expression analysis of $\alpha$-SMA and CD51 in PSCs isolated from pancreatic cancer. (A) CD51 mRNA expression levels in PSCs isolated from pancreatic cancers, their adjacent normal pancreas (nPSC), pancreatic cancer cell lines, and human normal pancreatic epithelial cells (NPE). CD51 mRNA expression was normalized by GAPDH expression. (B) Representative microphotographs of PSCs stained with anti- $\alpha$-SMA (red) and anti-CD51 (green) antibodies. DAPI (4',6-diamidino-2-phenylindole) (blue) was used for nuclear staining. CD51 was expressed in $\alpha$-SMA-positive PSCs. (C and D) CD51 mRNA (C) and protein (D) expression levels in PSCs were decreased by shRNA-mediated knockdown of CD51. "P<0.05. (E) Proliferation of PSCs was suppressed by CD51 knockdown. "P<0.05. (F) Migration of PSCs was suppressed by CD51 knockdown. ${ }^{*} \mathrm{P}<0.05$.

Knockdown of CD51 decreases the expression of ECM-related genes and tumor-stromal interaction-related genes in PSCs. One of the hallmarks of pancreatic cancer is an excessive amount of ECM known as desmoplasia, which is thought to be formed by PSCs activated by inflammation or carcinoma cells. We investigated whether CD51 was involved in the activation of PSCs and affected ECM-related gene expression. The gene expression of $\alpha$-SMA, which is a marker of acti- vated PSCs, was decreased by knockdown of CD51 (Fig. 3A). ECM-related genes, such as collagen I, fibronectin, periostin, and osteopontin, were all downregulated by knockdown of CD51 (Fig. 3A).

PSCs not only contribute to the formation of tumor desmoplasia but also enhance tumor malignancy by tumorstromal interactions. Therefore, we next examined the impact of CD51 on the expression of genes involved in 

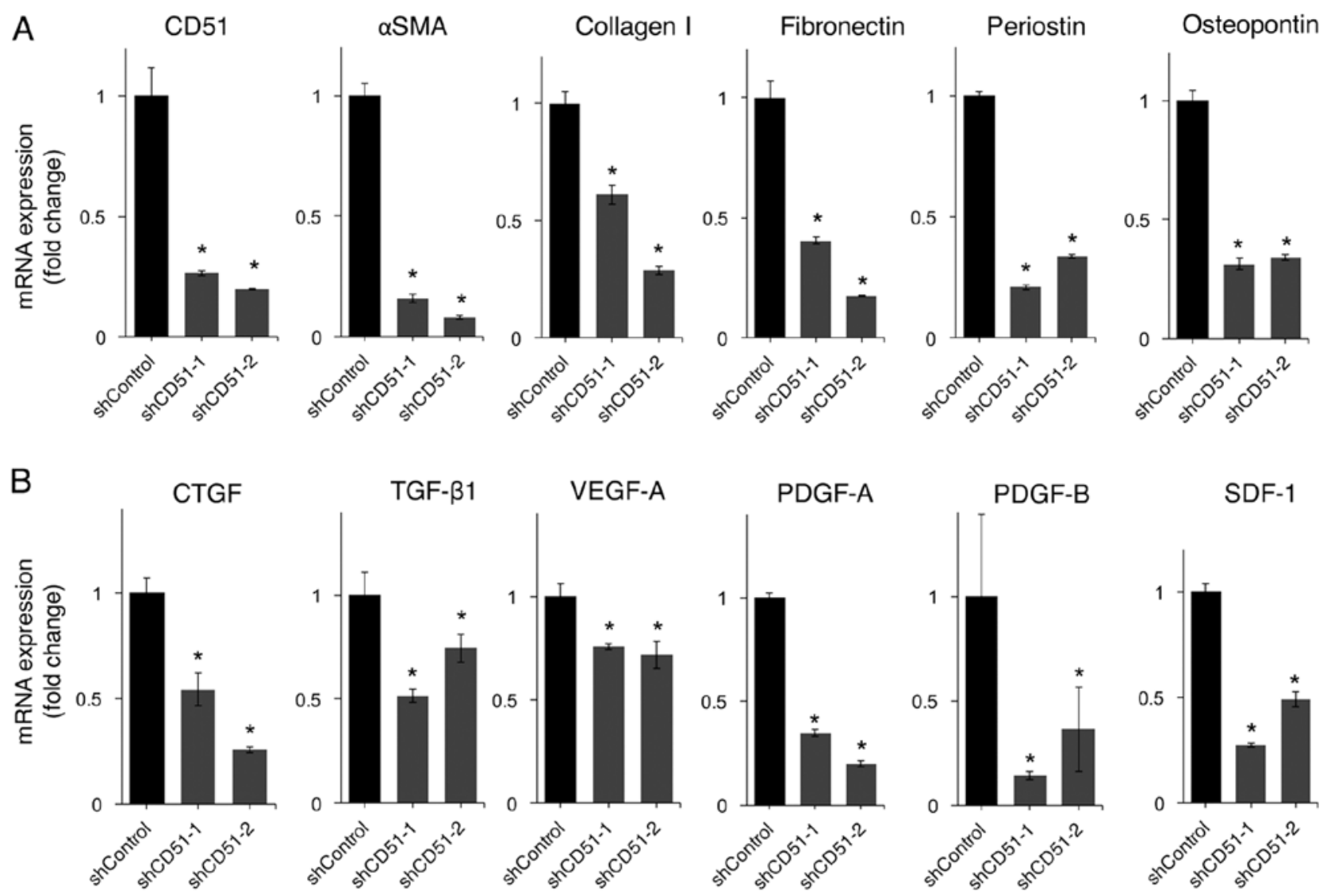

Figure 3. Effects of CD51 knockdown in PSCs on the expression levels of ECM-related genes and various growth factors associated with tumor-stromal interactions. (A) qRT-PCR showed that mRNA expression of ECM-related genes such as collagen I, fibronectin, periostin, and osteopontin were decreased by CD51 knockdown. $\alpha$-SMA, a marker for activation of PSCs, was also suppressed by CD51 knockdown. * P<0.05. (B) qRT-PCR showed that mRNA expression levels of some growth factors were decreased by CD51 knockdown. * $\mathrm{P}<0.05$.

tumor-stromal interactions. Gene expression levels of growth factors secreted by PSCs to stimulate pancreatic cancer cells to enhance malignancy, such as CTGF, TGF- $\beta 1$, VEGF-A, PDGF-A, B, and SDF-1, were all downregulated by knockdown of CD51 (Fig. 3B).

Knockdown of CD51 in PSCs reduces tumor growth, tumor stroma, and cancer cell proliferation in vivo. To analyze the effect of CD51 in PSCs on tumor growth in vivo, we transplanted SUIT-2 cells into the back of nude mice with or without immortalized PSCs that were transfected with control-shRNA (PSCs-shControl) or CD51-targeted shRNA (PSCs-shCD51). Tumor growth was more rapid in mice transplanted with PSCs-shControl than SUIT-2 cells alone. In mice transplanted with PSCs-shCD51, tumor growth was inhibited compared with those transplanted with PSCs-shControl (Fig. 4A and B). Tumor weights at 6 weeks after transplantation were also reduced when transfected with PSCs-shCD51 (Fig. 4C).

To elucidate the cause of the reduction of the mouse subcutaneous tumor, we first assessed the amount of tumor stroma, as PSCs produced ECM and knockdown of CD51 in PSC in vitro led to decreased expression of ECM-related genes (Fig. 3A). We performed Sirius red staining and immunohistochemistry for $\alpha$-SMA. Tumors transplanted with PSCs-shCD51 showed smaller Sirius red-positive areas and less $\alpha$-SMA-positive cells than those transplanted with PSCs-shControl, which were consistent with the in vitro data (Fig. 4D-F).
Since PSCs enhance tumor malignancy by interacting with cancer cells, we then investigated whether knockdown of CD51 in PSCs influenced cancer cell proliferation, resulting in tumor reduction. Immunohistochemistry for PCNA showed that cancer cell proliferation was inhibited in tumors transplanted with PSCs-shCD51 compared with those transplanted with PSCs-shControl (Fig. 4G and H). This result indicates that PSCs expressing CD51 enhance tumor proliferation by tumor-stromal interactions and that targeting CD51 would be an effective therapeutic option for pancreatic cancer.

\section{Discussion}

In the present study, we found that high expression of CD51 in pancreatic cancer stroma was associated with lymph node metastasis, positive pathologic margin, and poor patient survival. In addition, high expression of CD51 in cancer cells correlated with lymph node metastasis and G3 grade tumor, which is partly consistent with a previous report that showed an association between $\alpha v \beta 3$ integrin expression in pancreatic cancer and lymph node metastasis (25). To the best of our knowledge, our work provides the first findings of the relationship between stromal CD51 expression and poor patient survival in pancreatic cancer.

The stromal microenvironment plays an important role in cancer progression in pancreatic cancer. Activated PSCs are main components in tumor stroma that interact with cancer 
A

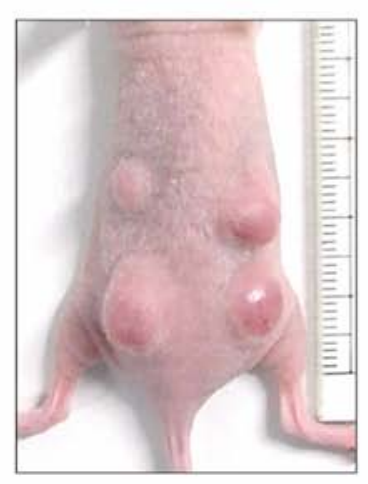

B

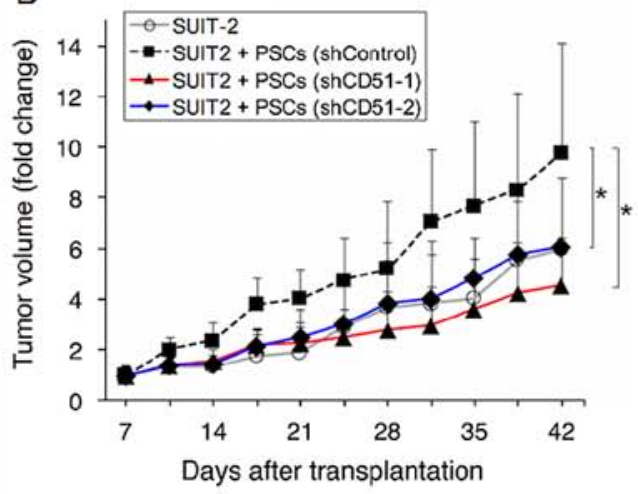

SUIT2 +

D
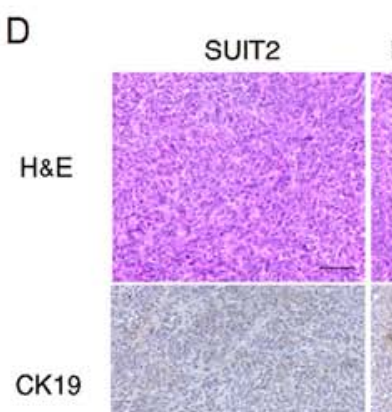

PSCs (shControl)

CK19

\section{to}

Sirius red

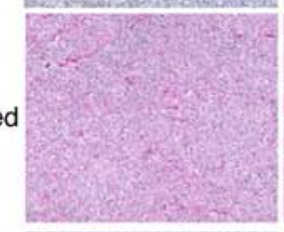

aSMA
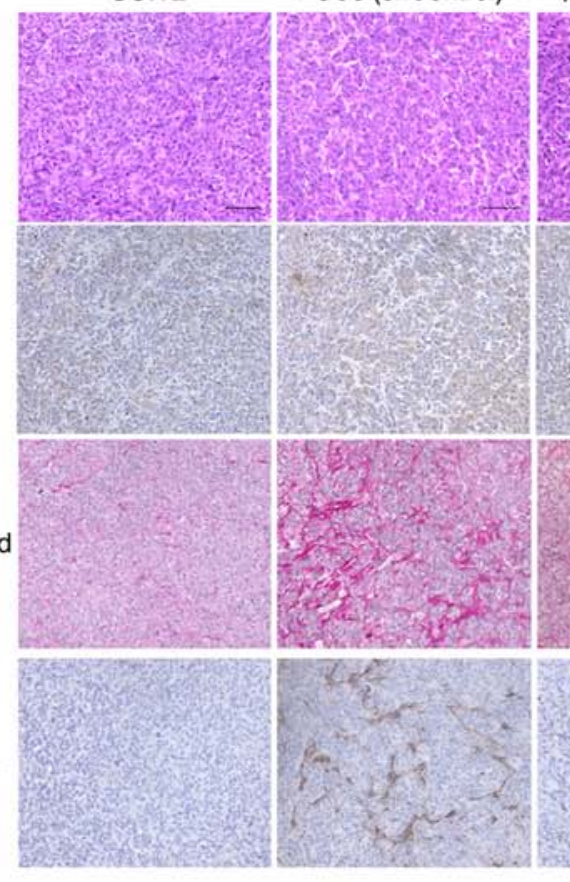

PSCs (shCD51-1)
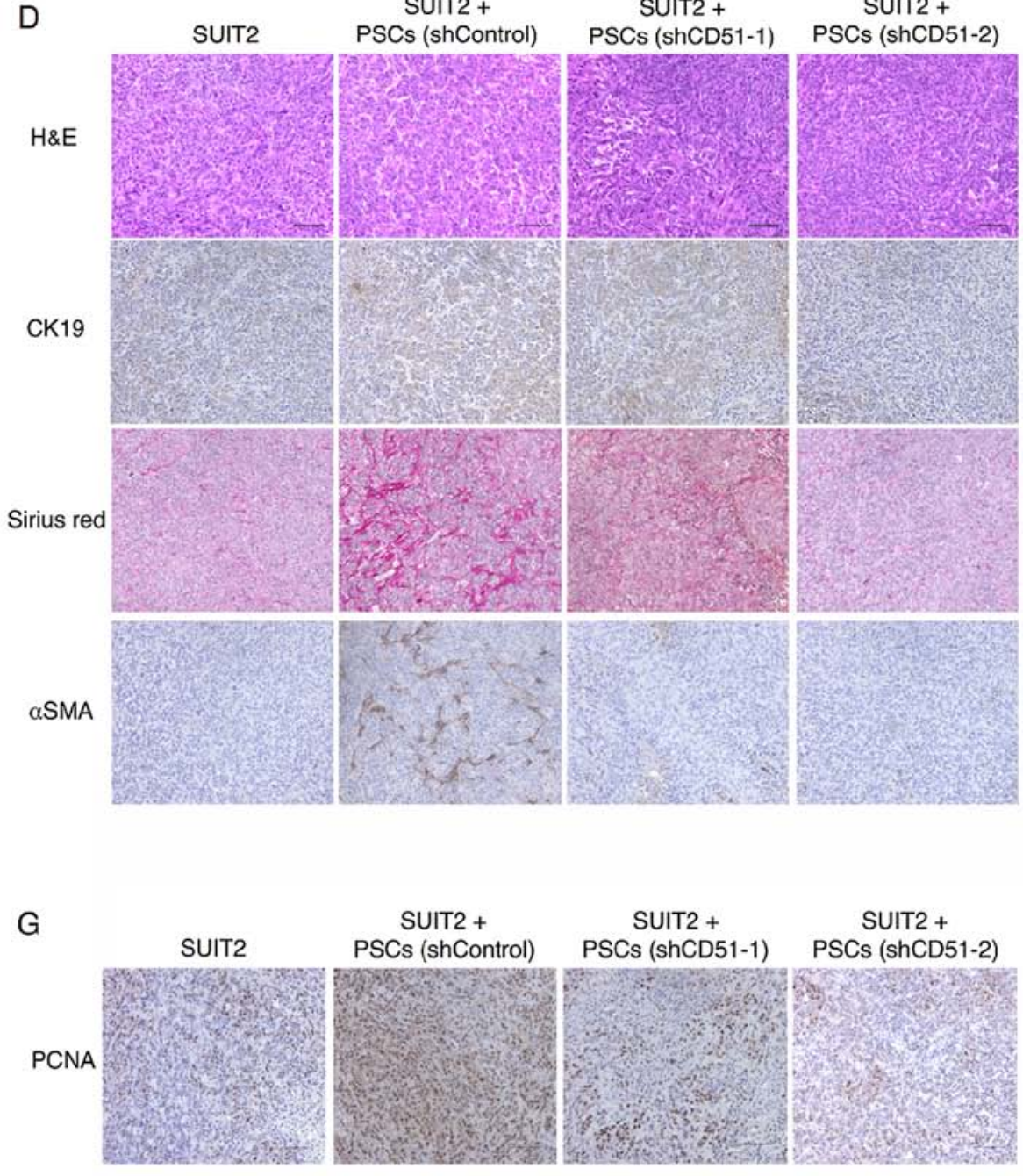
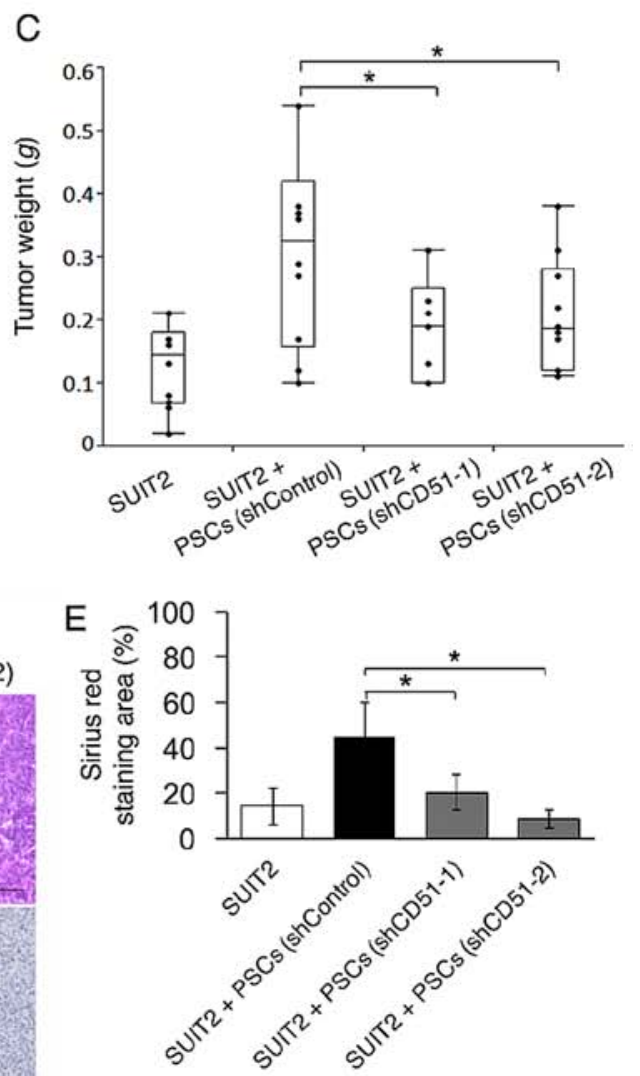

$F$ อ
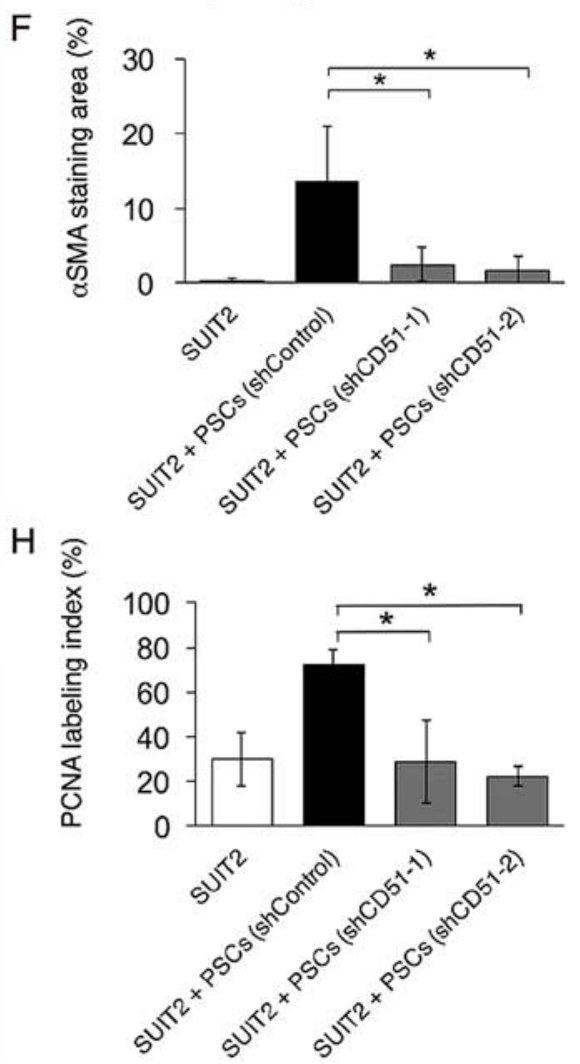

Figure 4. Effects of CD51 in PSCs on tumor growth in vivo. (A) SUIT-2 cells were transplanted subcutaneously in the back of nude mice with or without PSCs that were transfected with control-shRNA (shControl) or CD51-targeted shRNA (shCD51-1, 2). A representative image at day 35 is shown. Left upper, SUIT2; left lower, SUIT-2 plus PSCs (shControl); right upper, SUIT-2 plus PSCs (shCD51-1); right lower, SUIT-2 plus PSCs (shCD51-2). Tumor growth was inhibited by CD51 knockdown in PSCs. (B) Tumor volume was monitored twice a week from 1 week after transplantation up to day 42 . Relative tumor volume is shown. Data are shown as means+SD. ${ }^{*} \mathrm{P}<0.05$. (C) Tumors were excised on day 42 , and tumor weight was measured. Tumors transplanted with CD51-silenced PSCs showed lower weight. ${ }^{*} \mathrm{P}<0.05$. (D) Representative images of H\&E staining, Sirius red staining, and immunohistochemical staining for CK19 and $\alpha$-SMA in subcutaneous tumors. The amount of collagen fibers and $\alpha$-SMA-positive cells were reduced in tumors transplanted with CD51-silenced PSCs. (E and F) Quantification of Sirius red staining area (E) and $\alpha$-SMA-positive staining area (F). ${ }^{*} \mathrm{P}<0.05$. (G) Immunohistochemistry for proliferating cell nuclear antigen (PCNA). Proliferation of cancer cells is inhibited in tumors transplanted with CD51-silenced PSCs. (H) PCNA labeling index was calculated by dividing the number of PCNA-positive nuclei by total number of nuclei. " $\mathrm{P}<0.05$. 
cells to enhance malignancy. Interactions between PSCs and cancer cells are mediated by various soluble factors such as CTGF, PDGF, and SDF-1 (10,26,27). We showed that CD51 knockdown in PSCs led to downregulation of these factors in vitro as well as decreased proliferation of PSCs. These results may account for the suppression of cancer cell proliferation observed in vivo in transplantation of CD51-silenced PSCs. We also revealed the impact of CD51 in PSCs on the formation of desmoplasia. CD51 knockdown led to the inactivation of PSCs and decreased production of ECM, resulting in the reduction of tumor stroma. In addition, suppressed motility of PSCs by CD51 knockdown may also contribute to the reduction of stroma, as the migration of PSCs is likely to be needed for the formation of desmoplasia (28). Together with the suppression of cancer cell proliferation, these results yielded tumor volume reduction. As desmoplasia is a barrier to chemotherapeutic agents in delivery to cancer cells, further studies to elucidate whether targeting CD51 in PSCs augments conventional chemotherapy are needed.

Furthermore, some studies showed that cilengitide, a small molecule antagonist of integrin $\alpha v \beta 3$ and $\alpha v \beta 5$, was effective in the treatment of some solid tumors such as malignant glioma and laryngeal cancer $(23,29)$. In the present study, we focused on CD51 in PSCs, not cancer cells, and so the precise role of CD51 in pancreatic cancer cells was not completely elucidated. However, our immunohistochemistry results in pancreatic cancer tissues showed that CD51 was also highly expressed in cancer cells in $36 \%$ of the cases, indicating that targeting CD51 would also have direct effects on cancer cells. In fact, the motility of pancreatic cancer cells was inhibited by CD51 knockdown in vitro (data not shown). This result suggests that targeting CD51 may have an effect not only on PSCs but also on pancreatic cancer cells.

In conclusion, we demonstrated that CD51 expression in pancreatic cancer stroma was associated with enhanced tumor malignancy and that suppression of CD51 in PSCs inhibited tumor growth by reducing stroma and altering tumor-stromal interactions in pancreatic cancer.

\section{Acknowledgements}

This work was supported in part by JSPS Grant-in-Aid for Scientific Research (grant no. 26293305, 25293285, 25461024, 15K09046, 15H04933) and Scientific Research on Innovative Areas (grant no. 26108010).

\section{References}

1. Siegel RL, Miller KD and Jemal A: Cancer statistics, 2015. CA Cancer J Clin 65: 5-29, 2015.

2. Neesse A, Michl P, Frese KK, Feig C, Cook N, Jacobetz MA, Lolkema MP, Buchholz M, Olive KP, Gress TM, et al: Stromal biology and therapy in pancreatic cancer. Gut 60: 861-868, 2011.

3. Olive KP, Jacobetz MA, Davidson CJ, Gopinathan A, McIntyre D, Honess D, Madhu B, Goldgraben MA, Caldwell ME, Allard D, et al: Inhibition of Hedgehog signaling enhances delivery of chemotherapy in a mouse model of pancreatic cancer. Science 324: 1457-1461, 2009.

4. Provenzano PP, Cuevas C, Chang AE, Goel VK, Von Hoff DD and Hingorani SR: Enzymatic targeting of the stroma ablates physical barriers to treatment of pancreatic ductal adenocarcinoma. Cancer Cell 21: 418-429, 2012.
5. Moss RA, Moore D, Mulcahy MF, Nahum K, Saraiya B, Eddy S, Kleber M and Poplin EA: A Multi-institutional phase 2 study of imatinib mesylate and gemcitabine for first-line treatment of advanced pancreatic cancer. Gastrointest Cancer Res 5: 77-83, 2012.

6. Apte MV, Haber PS, Applegate TL, Norton ID, McCaughan GW, Korsten MA, Pirola RC and Wilson JS: Periacinar stellate shaped cells in rat pancreas: Identification, isolation, and culture. Gut 43: 128-133, 1998.

7. Bachem MG, Schneider E, Gross H, Weidenbach H, Schmid RM, Menke A, Siech M, Beger H, Grünert A and Adler G: Identification, culture, and characterization of pancreatic stellate cells in rats and humans. Gastroenterology 115: 421-432, 1998.

8. Bachem MG, Zhou S, Buck K, Schneiderhan W and Siech M: Pancreatic stellate cells - role in pancreas cancer. Langenbecks Arch Surg 393: 891-900, 2008.

9. Hwang RF, Moore T, Arumugam T, Ramachandran V, Amos KD, Rivera A, Ji B, Evans DB and Logsdon CD: Cancer-associated stromal fibroblasts promote pancreatic tumor progression. Cancer Res 68: 918-926, 2008.

10. Vonlaufen A, Joshi S, Qu C, Phillips PA, Xu Z, Parker NR, Toi CS, Pirola RC, Wilson JS, Goldstein D, et al: Pancreatic stellate cells: Partners in crime with pancreatic cancer cells. Cancer Res 68: 2085-2093, 2008.

11. Desgrosellier JS and Cheresh DA: Integrins in cancer: Biological implications and therapeutic opportunities. Nat Rev Cancer 10: 9-22, 2010.

12. Friedrichs K, Ruiz P, Franke F, Gille I, Terpe HJ and Imhof BA: High expression level of alpha 6 integrin in human breast carcinoma is correlated with reduced survival. Cancer Res 55: 901-906, 1995.

13. Bates RC, Bellovin DI, Brown C, Maynard E, Wu B, Kawakatsu H, Sheppard D, Oettgen P and Mercurio AM: Transcriptional activation of integrin beta6 during the epithelial-mesenchymal transition defines a novel prognostic indicator of aggressive colon carcinoma. J Clin Invest 115: 339-347, 2005.

14. Landen CN, Kim TJ, Lin YG, Merritt WM, Kamat AA, Han LY, Spannuth WA, Nick AM, Jennnings NB, Kinch MS, et al: Tumorselective response to antibody-mediated targeting of alphavbeta3 integrin in ovarian cancer. Neoplasia 10: 1259-1267, 2008.

15. Avraamides CJ, Garmy-Susini B and Varner JA: Integrins in angiogenesis and lymphangiogenesis. Nat Rev Cancer 8: 604-617, 2008.

16. Zhu CQ, Popova SN, Brown ER, Barsyte-Lovejoy D, Navab R, Shih W, Li M, Lu M, Jurisica I, Penn LZ, et al: Integrin alpha 11 regulates IGF2 expression in fibroblasts to enhance tumorigenicity of human non-small-cell lung cancer cells. Proc Natl Acad Sci USA 104: 11754-11759, 2007.

17. Taverna D, Moher H, Crowley D, Borsig L, Varki A and Hynes RO: Increased primary tumor growth in mice null for beta3 - or beta3/beta5-integrins or selectins. Proc Natl Acad Sci USA 101: 763-768, 2004

18. Jin H, Su J, Garmy-Susini B, Kleeman J and Varner J: Integrin alpha4beta1 promotes monocyte trafficking and angiogenesis in tumors. Cancer Res 66: 2146-2152, 2006.

19. Hynes RO: Integrins: Bidirectional, allosteric signaling machines. Cell 110: 673-687, 2002.

20. Ludbrook SB, Barry ST, Delves CJ and Horgan CM: The integrin alphavbeta3 is a receptor for the latency-associated peptides of transforming growth factors beta1 and beta3. Biochem J 369: 311-318, 2003.

21. Hinz B: It has to be the $\alpha v$ : Myofibroblast integrins activate latent TGF- $\beta 1$. Nat Med 19: 1567-1568, 2013.

22. Saalbach A, Wetzel A, Haustein UF, Sticherling M, Simon JC and Anderegg U: Interaction of human Thy-1 (CD 90) with the integrin alphavbeta3 (CD51/CD61): An important mechanism mediating melanoma cell adhesion to activated endothelium. Oncogene 24: 4710-4720, 2005.

23. Wang JT, Liu Y, Kan X, Liu M and Lu JG: Cilengitide, a small molecule antagonist, targeted to integrin alphanu inhibits proliferation and induces apoptosis of laryngeal cancer cells in vitro. Eur Arch Otorhinolaryngol 271: 2233-2240, 2014.

24. Ohuchida K, Mizumoto K, Murakami M, Qian LW, Sato N, Nagai E, Matsumoto K, Nakamura T and Tanaka M: Radiation to stromal fibroblasts increases invasiveness of pancreatic cancer cells through tumor-stromal interactions. Cancer Res 64: 3215-3222, 2004. 
25. Hosotani R, Kawaguchi M, Masui T, Koshiba T, Ida J, Fujimoto K, Wada M, Doi R and Imamura M: Expression of integrin alphaVbeta3 in pancreatic carcinoma: Relation to MMP-2 activation and lymph node metastasis. Pancreas 25: e30-e35, 2002.

26. Eguchi D, Ikenaga N, Ohuchida K, Kozono S, Cui L, Fujiwara K Fujino M, Ohtsuka T, Mizumoto K and Tanaka M: Hypoxia enhances the interaction between pancreatic stellate cells and cancer cells via increased secretion of connective tissue growth factor. J Surg Res 181: 225-233, 2013.

27. Gao Z, Wang X, Wu K, Zhao Y and Hu G: Pancreatic stellate cells increase the invasion of human pancreatic cancer cells through the stromal cell-derived factor-1/CXCR4 axis. Pancreatology 10 : $186-193,2010$.
28. Yang C, Zeisberg M, Mosterman B, Sudhakar A, Yerramalla U, Holthaus K, Xu L, Eng F, Afdhal N and Kalluri R: Liver fibrosis: Insights into migration of hepatic stellate cells in response to extracellular matrix and growth factors. Gastroenterology 124: 147-159, 2003.

29. Nabors LB, Mikkelsen T, Rosenfeld SS, Hochberg F, Akella NS, Fisher JD, Cloud GA, Zhang Y, Carson K, Wittemer SM, et al: Phase I and correlative biology study of cilengitide in patients with recurrent malignant glioma. J Clin Oncol 25: 1651-1657, 2007. 